\title{
Nanostructure of amorphous films
}

\author{
N.L. Dyakonenko ${ }^{1}$, V.A. Lykah ${ }^{2}$, A.V. Sinelnik ${ }^{3}$, I.A. Korzh ${ }^{4}$, V.I. Bilozertseva ${ }^{5}$ \\ National Technical University "Kharkiv Polytechnic Institute" \\ 2, Kirpichov str., 61002 Kharkiv, Ukraine, \\ E-mail:dnina490@gmail.coml,lykahva@yahoo.com²,

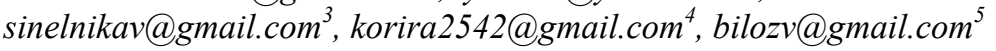

\begin{abstract}
The paper presents results of experimental and theoretical investigations of thin chalcogenide films at nanostructure level. Transmission electron microscopy demonstrated amorphous cluster structure. The equations for order parameters in cluster boundaries have been obtained and analyzed.
\end{abstract}

Keywords: chalcogenide thin film, nanocluster, cluster bond, order parameters, potential relief.

Manuscript received 13.01.17; revised version received 24.04.17; accepted for publication 14.06.17; published online 18.07.17.

\section{Introduction}

Amorphous chalcogenide films have a wide range of varying physical and chemical properties, which find applications in electronics and optoelectronics [1-5]. Functionality of thin films obtained from glassy materials often derives from their structure at different length scales. Nevertheless, the structural character investigations of these materials are carried out rarely without a significant opportunity to obtain systematic information. Most glasses have structures dominated by network in which bonding is predominantly covalent and have been described in terms of the random network model. Network glasses are the physical prototype for many self-organized systems, ranging from proteins to computer science. Cluster appearance in the noncrystalline objects is associated with partial selforganization of matter under chemical bonds influence $[6,7]$. There are general cluster formation conformities in different amorphous substances irrespective of their composition, preparation method, kind of conductivity and other properties. Conventional theories of gases, liquids, and crystals do not account the character of the glass-forming tendency, the phase diagrams of glasses [8] or their optimizable properties.
The degree of self-organization depends on preparation conditions (the substrate material and temperature). As the result, the breach of statistically homogeneous atomic distribution of nanosized irregularities (clusters) appears. In contrast to the crystalline state characterized by long-range order (LRO), i.e., by existence of correlations between the positions of every two atoms situated as far as possible one from another, the non-crystalline state is characterized by the absence of the LRO. The remnant is not total disorder but a certain limited order called as short-range order (SRO) defined by the inter-atomic correlations in the first coordination spheres of an arbitrary atom, i.e., up to the maximum distance where the bonding forces are active. In chalcogenide glasses, the order extends up to interatomic distances. On this basis, a new type of order was defined: the mediumrange order (MRO). It is known that glasses and liquids exhibit the first sharp diffraction peak that is evidence for intermediate range order caused by regularities in the packing of structural units.

This communication presents results of experimental and theoretical investigations of thin amorphous films of different chalcogenide systems at nanostructural level. 


\section{Experimental results}

The thin films of chalcogenide glass compounds $\mathrm{A}^{1} \mathrm{BiC}^{\mathrm{V} 1}\left(\mathrm{~A}^{1}-\mathrm{Li}, \mathrm{K}, \mathrm{Na}, \mathrm{Rb}, \mathrm{C}^{\mathrm{V} 1}-\mathrm{S}, \mathrm{Se}\right)$ were evaporated from the resistant-heated tungsten boat onto a relatively cold glass substrate $(300 \mathrm{~K})$ in vacuum $1 \mathrm{mPa}$. The results of TEM investigations are represented in Fig. 1. All these chalcogenide films have an amorphous structure. Amorphous layers have not an atomic-smooth surface, but consist of clusters from 5 to $15 \mathrm{~nm}$ in size. The contrast on the electron-microscopic images of clusters is supposed to be caused by material density changes. The absence of contact between clusters is their specific feature. There are regions of low density between clusters 1-2 $\mathrm{nm}$ in size for different films. Distribution of the relative frequency $N$ of clusters (a) and cluster boundaries (b) dimensions for $\mathrm{KBi}_{3} \mathrm{~S}_{5}$ films are shown in Fig. 2.

In the ideal crystal, chalcogen compounds form a plane triangular subgrate with the six-coordinated covalent bond. These planes interchange with bismuth layers (semimetal) and alkali that have the metal bond. That is, in the ideal crystal $\mathrm{A}^{1} \mathrm{BiC}^{\mathrm{V} 1}$ chalcogen layers with covalent directional bonds are interchanged with $\mathrm{Bi}$, alkali metallic (isotropic) bonds. The bond between layers does not seem to be covalent and metallic. It may be, to a large degree, ionic and unidirectional.

When condensing, the atoms are deposited in a uniform layer, in which they are placed in disorder, the stoichiometry of the compound being saved. As the result of disturbing the atom order, the lengths and orientation of chemical bonds are broken.

In low temperature experiments, atoms solidify in random positions. Homogeneous amorphous phase with full disordering the covalent bond lengths and orientations is realized through the homogeneous inversion of the covalent, metallic and ionic bonds order.

If the substrate temperature is high, the diffusion of atoms and crystal phase formation during deposition process are possible. In this way, the atomic system energy is minimized.

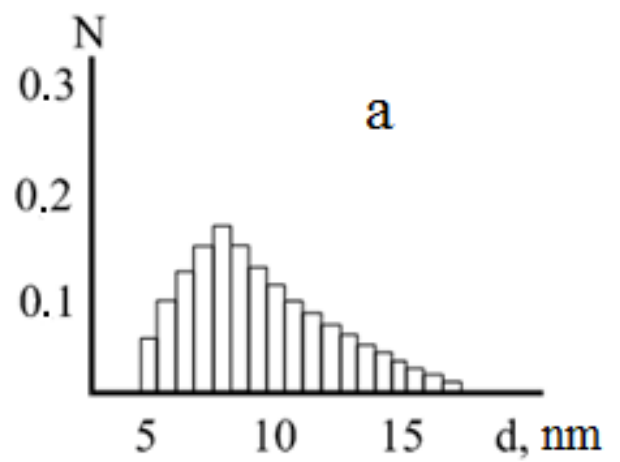

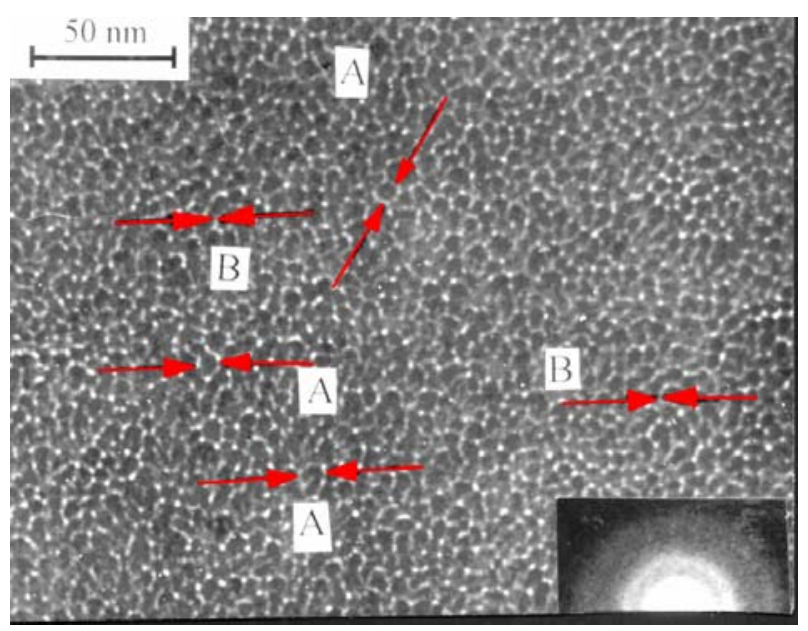

Fig. 1. Structure of $\mathrm{KBi}_{3} \mathrm{~S}_{5}$ films: thickness $h=40 \mathrm{~nm}$, vacuum level $P=10^{-3} \mathrm{~Pa}$, rate of condensation $0.1-0.5 \mathrm{~nm} / \mathrm{s}$, substrate temperature $T_{s}=300 \mathrm{~K}$. A - clusters, B - cluster boundaries.

In our experiments, the substrate temperature was less than the temperature of crystallization, that is the atoms order changing processes were not realized. But it was formed the structure with interchanging regions of low and high density of the film. The present measurements agree satisfactorily with following the theoretical analyses.

\section{Theoretical analysis}

Two mutually related order parameters have been chosen for description of an amorphous film structure: the mean deviation of angle from the optimal average value in covalent bond $\varphi$ and the average interatomic distance $r$. The spatial change of the order parameters is assumed to be slow.

There are various microscopic descriptions of interatomic interaction: Morse potential, model potential for Van der Waals and Lenard-Jones type interactions.

Parameter $\varphi$ should well describe bad ordering. The

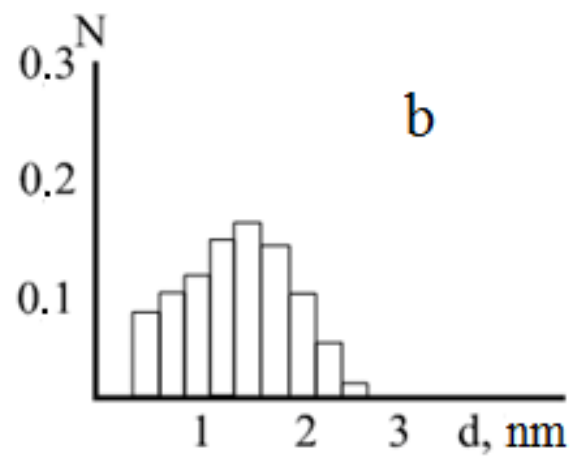

Fig. 2. Distribution of the relative frequency $N$ of cluster (a) and cluster boundaries (b) dimensions for $\mathrm{KBi}_{3} \mathrm{~S}_{5}$ films. 
sharp orientation of the bond and their periodicity, apparently, are well described by elliptic functions. The most sharp orientation among elliptic function is elliptic cosine $\operatorname{cn}(x, k)$, where $k$ is the elliptic module, $x$ is variable. In the limit case $k=0$, elliptic functions turn in trigonometrically $\sin x, \cos x$, and at $k \rightarrow 1$ they turn in hyperbolic ones: $\operatorname{sn}(x, k) \rightarrow \tanh (x), \operatorname{cn}(x, k) \rightarrow 1 / \cosh (x)$ [9].

The free energy density of the system $w=W / V=w_{\text {inh }}+w_{\text {hom }}$ consists of homogeneous $w_{\text {hom }}$ and inhomogeneous $w_{\text {inh }}$ parts. Let's write down the free energy density with the obvious account of angular dependence

$$
\begin{aligned}
& w_{\text {inh }}=\frac{E}{2}\left(\frac{\partial x}{\partial z}\right)^{2}+\frac{G l^{2}}{2}\left(\frac{\partial \varphi}{\partial z}\right)^{2}, \\
& w_{\text {hom }}=-\frac{a-b \cdot \operatorname{sn}\left(\chi-\chi_{0}\right)}{x^{n}}+\frac{c}{x^{n+m}},
\end{aligned}
$$

where $x$ is the average interatomic distance. The letters $a, b, c$ stand for average energy parameters of microscopic potential of interaction, they depend on average quantity of neighbours with different type of bound and effective radius. $E, G$ are effective Young and shear modules and $l$ is the effective length for angular deformation; $z$ is the coordinate along which there are changes of parameters of the system. $\varphi$ is the average angle of the bond calculated from an optimal value $\Phi_{0}$, $\varphi=\Phi-\Phi_{0}$. The angle is also normalized by period: $\chi_{0}=\Phi_{0} / k d=0,2 K, 4 K, \ldots$ the elliptic function period $4 K(k)$, where $K(k)$ is the full elliptic integral of the first kind. $\operatorname{sn}(\Phi / k d, k)$ is the Jacobi elliptic function, it sets periodicity of the bound change depending on the angle $\Phi$. $k$ is the elliptic module, $d-$ angular period of covalent bond (in section).

The sharp orientation of the covalent bond falls with increase in the distance and ionic rate [6]. The repulsion of atomic shells dominates at strong approach between them. According to it, we shall choose the elementary function having a maximum at the optimum bond length $r_{c}$. So, $1 / x^{n}$ and $1 / x^{n+m}$ are contributions to potential of Lenard-Jones type. The covalent bond is directed and strong. The sharp minimum of potential corresponds to $\operatorname{sn}^{2}(\Phi / k d, k)=0$.

With increasing the interatomic distance, the ionic component of the bond increases, the depth of potential decreases. It corresponds to $\operatorname{sn}^{2}(\Phi / k d, k)=1$.

The free energy variation with the order parameters leads to the system of Lagrange-type equations:

$$
\left\{\begin{array}{l}
\frac{\partial}{\partial z} \cdot\left(\frac{\partial w}{\partial r^{\prime}}\right)-\frac{\partial w}{\partial r}=0 \\
\frac{\partial}{\partial z} \cdot\left(\frac{\partial w}{\partial \varphi^{\prime}}\right)-\frac{\partial w}{\partial \varphi}=0
\end{array}\right\}
$$

Here and somewhere further, $r=x$. The integral of the equations' system is:

$$
\frac{E}{2}\left(\frac{\partial x}{\partial z}\right)^{2}+\frac{G l^{2}}{2}\left(\frac{\partial \varphi}{\partial z}\right)^{2}-\left[-\frac{a-b \operatorname{sn}^{2} \chi}{x^{n}}+\frac{c}{x^{n+m}}\right]=C
$$

The homogeneous part is the effective "potential energy" (Fig. 3) of the system. Let's introduce dimensionless parameters: $x=\gamma r_{c}, \beta=b / c$, where

$r_{c}=x_{0}=\sqrt[m]{\frac{(n+m) c}{a_{n}}}$

is the equilibrium distance for covalent bond at $\mathrm{sn}^{2}(\Phi / k d, k)=0$;

$a=\frac{(n+m) c}{n r_{c}^{m}}, E r_{c}^{2}=\frac{r_{c}^{m+n}}{c}=l_{r}^{2}, G l^{2}=\frac{r_{c}^{m+n}}{c}=l_{\varphi}^{2}$,

$\varsigma=\frac{z}{l_{r}}$

Then, the integral in dimensionless variables will become

$$
\begin{aligned}
& \frac{1}{2}\left(\frac{\partial \gamma}{\partial \varsigma}\right)^{2}+\frac{\lambda^{2}}{2}\left(\frac{\partial \varphi}{\partial \varsigma}\right)^{2}- \\
& -\left[-\frac{(n+m)\left(1-\beta \operatorname{sn}^{2} \chi\right)}{n \gamma^{n}}+\frac{1}{\gamma^{n+m}}\right]=C .
\end{aligned}
$$

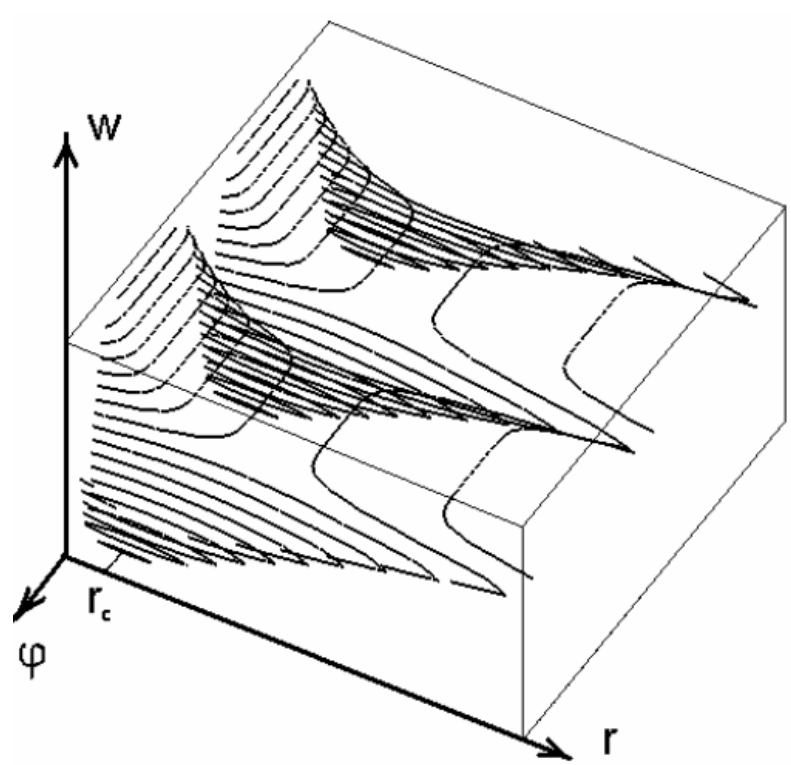

Fig. 3. Potential relief for the density of free energy $w . \varphi$ is the average angle of covalent bond, $r=x$ - average length of the bonds between atoms, $r_{c}$ corresponds to the minimum energy within clusters. Different minima correspond to different clusters. Level lines are shown. 
Boundary conditions in domains are: $\operatorname{sn}^{2}(\Phi / k d, k)=0, \quad \gamma^{\prime}=0, \quad \varphi^{\prime}=0 \quad\left(\gamma=1, \quad x=r_{c}\right), \quad$ it defines the integral value

$$
\frac{1}{2}\left(\frac{\partial \gamma}{\partial \zeta}\right)^{2}+\frac{\lambda}{2}\left(\frac{\partial \varphi}{\partial \zeta}\right)^{2}+\frac{(n+m)\left(1-\beta \mathrm{sn}^{2} \chi\right)}{n \gamma^{n}}-\frac{1}{\gamma^{n+m}}-1=0
$$

$\gamma(\varsigma), \varphi(\varsigma)$ - (functions) dimensionless parameters of interatomic distance and the bond angle (parameters of interatomic interaction); $n, m$ are powers in the potential of Lenard-Jones type; $\beta$ is the parameter responsible for depth of modulation of covalent bond; $\lambda$ - parameter equal to the relation of the angle and length scales of the bond.

It is obvious that the system is transformed into a state with reoriented bonds and decreased their average lengths to minimize energy. In a three-dimensional case, this process, probably, is possible with reduction of total volume of a sample. Under condensation on a substrate for strong connection of the sample atoms with a substrate, the average (not reoriented) values of the bond lengths should be kept, and after formation of several layers their local reorientation is possible. Qualitatively, this process is similar to that of formation of discrepancy dislocations. The experiment shows saving the initial stratified structure for the films, which thickness is larger than the average cluster sizes.

To considerate spatial changes of the order parameters (average interatomic distance $r$ and average angle $\varphi$ ), we have assumed their slow change in space. The problem of the variables splitting is old and hard [10]. Here, we choose a rectangle way. This path is shown in Fig. 4, it goes between two potential minima $(a, e)$ that correspond to two neighbor clusters. Besides, it requires the energy minimum (saddle point $c$ ) at the maximum interatomic distance. So, the path has two regions where only the distance changes $(a-b$ and $d-e)$ and one region where only the angle changes $(b-c-d)$. Boundary conditions for single boundary between clusters (point 0 corresponds to 2K in Figs. 3 and 4):

$z= \pm \infty, \frac{\partial \varphi}{\partial z}=0, \varphi= \pm \frac{d}{2}$.

The choice of an integration contour, i.e., transfer from one domain (cluster) to another, splits variables $x=r$ and $\varphi$. Optimization of the domain boundary depends on choice of an integration contour. For variables splitting, we'll choose a contour shown in Figs. 4 and 5. The coordinate dependence of the angle distance breaks up into two sites. This contour corresponds to a rigid angular dependence of potential and soft dependence of the interatomic distance.

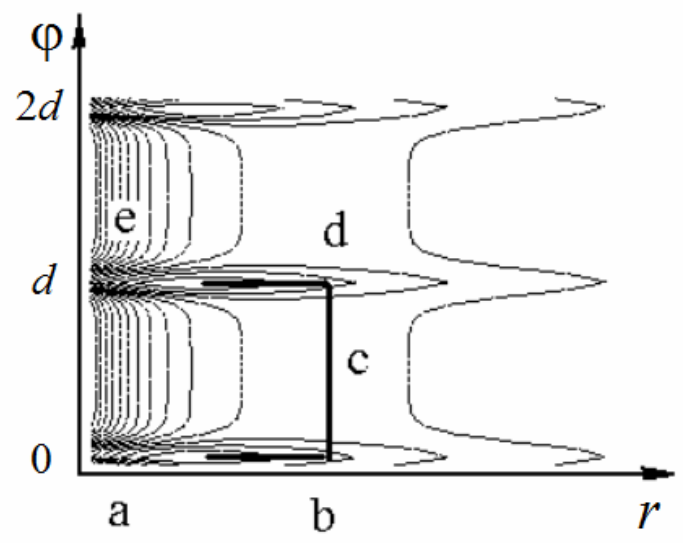

Fig. 4. The levels of the potential $w(r, \varphi)$ at $r$ - $\varphi$ plane (compare with Fig. 3). Solid line with straight parts is the integration pathway through a cluster boundary on the $r-\varphi$ plane.

At the first step, let us consider the change of bond angles at the fixed bond lengths $r=r_{c s}=$ const, which corresponds to the saddle point $c$ of the effective potential. Figs. 4 and 5 show the integration path along the $r-\varphi$ plane and in $r-w$ cross-section as well as the saddle point $c$ at $\varphi=K$ and $r=r_{c s}$. Then, the latter integral of the equation will have a form:

$\frac{\lambda^{2}}{2}\left(\frac{\partial \varphi}{\partial \varsigma}\right)^{2}-\frac{(n+m) \beta \operatorname{sn}^{2} \chi}{n \gamma^{n}}=C$

Variable data are separated, and the solution can be obtained by integration

$$
\int d \varsigma=\varsigma-\varsigma_{0}=\int C_{2} \frac{d \varphi}{\sqrt{C_{1}-\operatorname{sn}^{2}(\varphi, K)}}
$$

where functions $C_{1}, C_{2}$ depend on parameters $r_{c s}, \beta, \gamma, \lambda$, $m, n$. The obvious coordinate dependence of the angle $\varphi(z)$ is shown in Fig. 5 as the curve $b-c-d$.

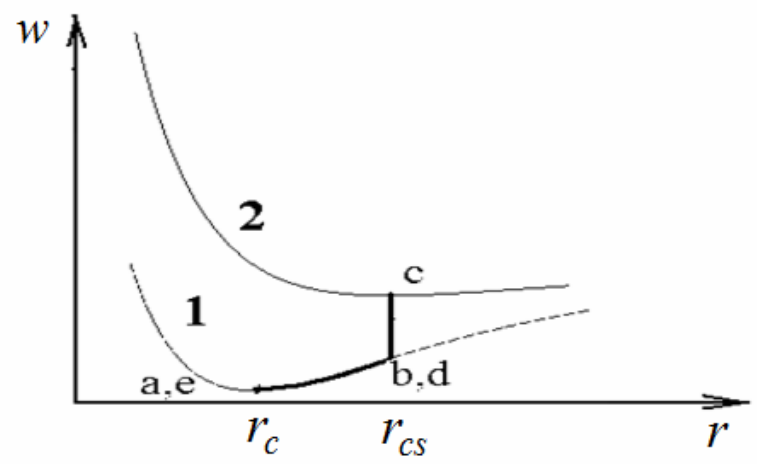

Fig. 5. Curve 1 is cross-section of $w(r, \varphi)$ at $\varphi=0$; i.e. $\operatorname{sn}(\varphi)=0$ (absolute min). Curve 2 is the cross-section of $w(r, \varphi)$ at $\varphi=K(k)$, i.e., $\operatorname{sn}(\varphi)=1$. Thick line is the path for the cluster boundary on the $r-\varphi$ plane. Letters $a-e$ correspond to the same points in Fig. 4. 


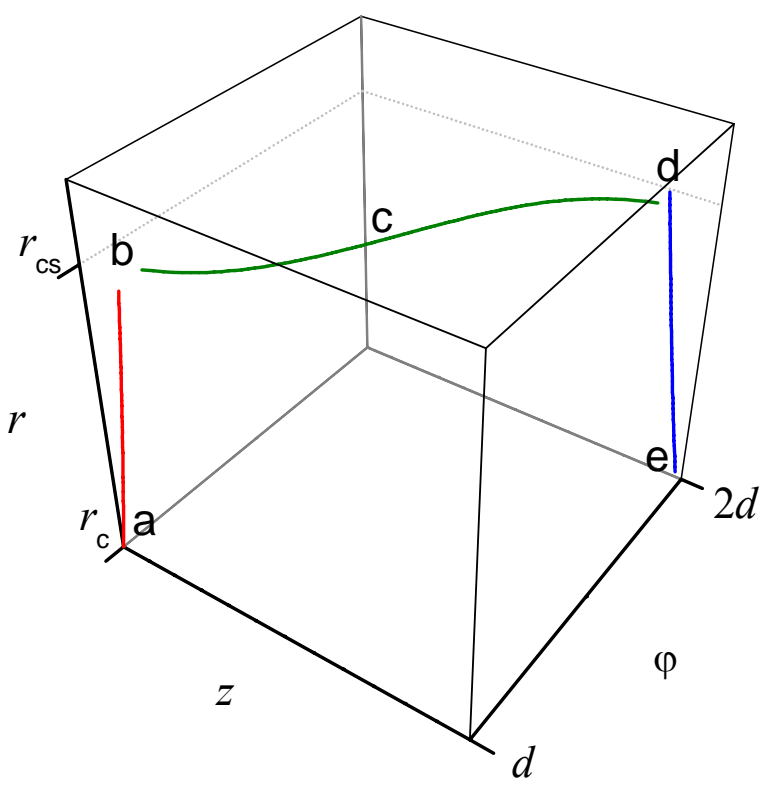

Fig. 6. Change of the parameters $r, \varphi$ in dependence on the space coordinate $z$. Letters and points $a-e$ correspond to the same objects in Figs. 4 and 5. The points $b$ and $d$ need tailoring because of break shape of the path.

At the second step, let us consider the bond length change in the range $\left[r_{c}, r_{c s}\right]$ at the fixed angles bond $\varphi=0,2 K$. Integration and the coordinate dependence of the bond length $r(z)=x(z)$ are obtained using the same way as for the angle. The obvious coordinate dependence of the bond length is shown in Fig. 5, 6 as the line segments $a-b$ and $d-e$.

The cluster boundary gives positive contribution to the general energy (see Fig. 5). The found long bonds inside the cluster boundaries correspond to lower density and the experimentally found better electron transition in TEM. Clusters have negative contribution to the energy of an initial strongly disordered system. The reason is that the energy level decreases, which is caused by some reorientation and shortening the covalent bonds inside the clusters.

\section{Conclusions}

The free energy that describes atomic interaction in disordered state was introduced. The order parameters, angles and lengths of covalent bonds were introduced. The equations for cluster boundary were derived. The dependence of the order parameters on coordinates within the cluster boundaries was found. TEM results are found to be in reasonable agreement with theoretical estimates. The cluster is noncrystalline due to disordered atomic bonds with different lengths and angles. The cluster boundaries contain enlarged disordered bonds.

\section{References}

1. Brodsky M. Amorphous Semiconductors, in: Topics in Applied Physics. Vol. 36. Berlin: Springer-Verlag, 1979.

2. Bilozertseva V.I., Khlyap H.M., Shkumbatyuk P.S., Dyakonenko N.L., Mamaluy A.O., Gaman D.O. LiBe-Se semiconductor thin films: technology, structure and electrophysical properties. Semiconductor Physics, Quantum Electronics \& Optoelectronics, 2010. 13, No. 1. P. 61-64.

3. Bogoslovskiy N.A., Tsendin K.D. Physics of switching and memory effects in chalcogenide glassy semiconductors. Semiconductors. 2012. 46, No. 5. P. 559-590.

4. Paiuk A.P., Stronski A.V., Vuichik N.V., Gubanova A.A., Kryskov Ts.A., Oleksenko P.F. Mid-IR impurity absorption in $\mathrm{As}_{2} \mathrm{~S}_{3}$ chalcogenide glasses doped with transition metals. Semiconductor Physics, Quantum Electronics \& Optoelectronics. 2012. 15, No. 2. P. 152-156.

5. Vakiv M., Golovchak R., Chalyy D., Shpotyuk M., Ubizskii S., Shpotyuk O. Peculiarities of valence band formation in As-Ge-Se semiconductor glasses. Semiconductor Physics, Quantum Electronics \& Optoelectronics. 2012. 15, No. 1. P. 32-34.

6. Dembovsky S.A., Chechetkina E.A. Glassy materials clarified through the eyes of hypervalent bonding configurations. J. Optoelectron. Adv. Mater. 2001. 3, No. 1. P. 3-18.

7. Phillips J.C. Chemical bounding and heats of formation in chalcogenide network compounds $\mathrm{As}_{2}(\mathrm{~S}, \mathrm{Se})_{3}$ and $\mathrm{Ge}(\mathrm{S}, \mathrm{Se})_{2}$. Phys. Rev. 1983. 28. P. 28-29.

8. Lazarev V.B., Salov A.V., Berulia S.I. Ternary Compound of Semiconductor Systems $\mathrm{A}^{\mathrm{I}} \mathrm{B}^{\mathrm{V}} \mathrm{C}^{\mathrm{VI}}$. Moscow: Nauka, 1982 (in Russian).

9. Dwight H.B. Tables of Integrals and Other Mathematical Data. 4-th Edition. New York: Macmillan, 1961.

10. Rajaraman R. Solitons and Instantons: An Introduction to Solitons and Instantons in Quantum Field Theory. North-Holland, Amsterdam, 1984. 